\title{
Canadian Association of Neuropathologists Abstracts of papers and cases presented at the 41st Annual Meeting
}

\author{
September 13-15, 2001
}

Montreal, Quebec

Can. J. Neurol. Sci. 2001; 28: 369-375

The Forty-First Annual Meeting of the Canadian Association of Neuropathologists was held from September 1315, 2001 at the Fédération des Médecins Spécialistes du Québec in Montreal, Quebec. Local arrangements were coordinated by Dr. Steffen Albrecht.

The scientific sessions were comprised of 20 platform presentations and 10 diagnostic case presentations, organized under the following headings: Epilepsy and pediatric/ developmental disorders; Dementia and degenerative processes; Infection and inflammatory processes; Tumors and miscellaneous disorders. The Royal College of Physicians and Surgeons of Canada speaker was Dr. Jean-Pierre Julien, Ph.D., Professor, Department of Neurology and Neurosurgery, and Medicine, McGill University. His lecture was entitled "New Insights into the Pathogenesis of Amyotrophic Lateral Sclerosis". The Jerzy Olszewski Lecture was presented by Dr. Andrea LeBlanc, Associate Professor, Department of Neurology and Neurosurgery, McGill University. Dr. LeBlanc's presentation was entitled "Prion Protein Protects Human Neurons Against Apoptosis."

\section{PLATFORM PRESENTATIONS}

\section{Bilateral Rasmussen's encephalitis: Post-mortem documentation in a five-year-old}

\section{S.M. TOBIAS, Y. ROBITAILLE, W.F. HICKEY, C.H. RHODES, R. NORDGREN and F. ANDERMANN (Dartmouth-Hitchcock Medical Center, New Hampshire (S.M.T., W.F.H., R.N., C.H.R.), Montreal Neurological Hospital and Institute (F.A.), the Hôpital Sainte-Justine, Montreal (Y.R.)}

A case of Rasmussen's disease with bilateral involvement in a five-year-old girl is reviewed. Rasmussen's disease is a rare progressive encephalitis that causes intractable seizures, cognitive decline, and inflammatory changes in brain. The neurological involvement is characteristically unilateral. Bilateral involvement in this case was suspected within five months of presentation and was confirmed by bilateral frontal lobe biopsies. The severity and progression of the disease in this case was remarkable and resulted in the patient's death by age five, despite numerous attempted therapeutic interventions. Autopsy findings confirmed the diagnosis of Rasmussen's encephalitis with bilateral involvement.

\section{Balloon cells in different pathological entities}

K. MEAGHER-VILLEMURE ${ }^{1}$, S. GEBHARD ${ }^{1}, J-G$. VILLEMURE $^{2}$ (Institut Universitaire de Pathologie ${ }^{1}$, Service de Neurochirurgie ${ }^{2}$, Centre Hospitalier Universitaire Vaudois, Lausanne, Switzerland)

Morphological studies of resected tissues for the treatment of intractable seizures reveal a large spectrum of anomalies. Malformations, vascular lesions, infectious or scarring processes, slow growing tumors are the most frequently encountered findings. Balloon cells are well known to occur in some dysplastic lesions and are characteristic morphological features of the tuber in tuberous sclerosis of Bourneville. These cells are often one type of the abnormal cellular components found in epileptic material. We describe the presence of balloon cells as being the main histological abnormality in different clinical conditions such as cortical dysplasia, hemimegalencephaly, dysplasia mimicking tumor, and compare their morphology specimens from tuberous sclerosis. Little is known yet as to the etiology of these cells. TSC2 tuberin is expressed in cell processes and cell bodies of the balloon cells in tuber of tuberous sclerosis and weakly in the ones from the white matter. In cortical dysplasia, this expression is not seen in the balloon cells. A variety of markers, features of immaturity, have been identified in the balloon cells of cortical dysplasia like MAP2c, Map 1b, Nestin and Map 2b. Different markers implicated in the differentiation pathways have also shown variable expression. We studied the presence of balloon cells in different clinical 
conditions with emphasis on their morphology, immunohistochemistry, ultrastructure features in comparison to the balloon cells present in tuber or subependymal giant cells tumor of tuberous sclerosis.

\section{Neuropathological changes in a murine model of progressive myoclonus epilepsy: Cystatin B deficiency and Unverricht-Lundborg disease}

\section{P. SHANNON ${ }^{1}$, L.A. PENNACCHIO ${ }^{2,3}$, B. MINASSIAN ${ }^{4}$, and} R.M. MYERS ${ }^{2}\left({ }^{1}\right.$ Department of Pathobiology and Laboratory Medicine, The University of Toronto, and the Toronto Western Hospital. Toronto Ontario, Canada ${ }^{2}$ Department of Genetics, School of Medicine, Stanford University, Stanford, CA ${ }^{3}$ Genome Sciences Department, Lawrence Berkeley National Laboratory, ${ }^{4}$ Department of Neurology, The Hospital for Sick Children, Toronto, Ontario, Canada)

Progressive myoclonus epilepsy of the Unverricht-Lundborg type (EPM1) is a recessively inherited neurodegenerative disease caused by loss-of-function mutations in the human cystatin B gene. Recently, mice lacking this gene have been described and display myoclonic seizures, progressive ataxia and cerebellar pathology closely paralleling EPM1 in humans. To provide further insight into our understanding of EPM1, we report pathological findings in brains from cystatin B-deficient mice. In addition to confirming the loss of cerebellar granular cell neurons by apoptosis, we identified areas of gliosis and decreased neuronal density, most marked in the presubiculum, and parasubiculum of the hippocampal formation, as well as the entorhinal cortex, neocortex and striatum. Furthermore, widespread white matter gliosis was also noted, which may be a secondary phenomenon. Although neuronal density was mildly reduced in the cerebral cortex, cellular atrophy is a prominent finding, which we demonstrate in the superficial neurons of the prosubiculum. We conclude that neuronal atrophy is an important consequence of loss of cystatin-B function, which suggests a physiological role for this protein in the maintenance of normal neuronal structure.

\section{Post-operative prognosis after long term follow up in childhood temporal lobe epilepsy: Neuropathologic correlates}

Y. ROBITAILLE, C. BOCTI, J. MICHAUD, A. BOUTHILLIER, L. CARMANT (Depts. of Pathology \& Cell Biology, Neurology and Neurosurgery, University of Montreal, Montreal, QC.)

We reviewed the clinical records of 22 pediatric patients who had temporal lobectomy for drug resistant seizures between 1979-1999. All had partial-complex seizures, $48 \%$ with secondary generalization. Prior to surgery, seizures occurred daily in $69 \%$, with auras in $29 \%$. One child had atypical febrile seizures. The mean age of onset for seizures was $3.8 \pm 3.3$ years (range $=1 \mathrm{~m}-10$ years). All but two subjects had "en bloc" resections, and there were two lesionectomies. The postoperative follow-up range was four months -19 years $($ mean $=$ $6.5 \pm 4.2$ years). A blind review of neuropathologic data was performed (YR). Cortical dysplasia was observed in 14 cases, with limbic extension in two, and mesial temporal sclerosis (MTS) in three. Only two patients had isolated MTS. There were two benign lateral temporal tumors (ganglioglioma $=1$, glioneuronal = 1), one of which was complicated by MTS and recurred. Postoperative results were: seizure free: $48 \%(10 / 21)$, $90 \%$ seizure frequency decrease: $29 \%(6 / 21),>50 \%$ seizure frequency decrease: $14 \%(3 / 21)$. Two patients failed to improve at all, one was lost to follow-up. We conclude that surgery affords substantial improvement of seizure control even to children with extensive cortical dysplasia. Moreover, as previously observed, temporal lobe epilepsy in childhood appears to be predominantly associated with cortical developmental pathology, more frequently with "double lesions" than adults.

\section{Microarray analysis of hydrocephalus in young and adult rats}

\section{JANANI BALASUBRAMANIAM and MARC R. DEL BIGIO}

(Department of Pathology, University of Manitoba, Winnipeg, Canada)

Hydrocephalus is associated with gradual progressive impairment and destruction of cerebral axons and neurons. To provide a comprehensive analysis of secondary changes in gene expression due to experimental hydrocephalus we used microarray-screening technique representing approximately 5000 genes. Hydrocephalus was induced in three-week-old and 8-10-week-old rats by injection of kaolin into cisterna magna. Brain tissues were examined 21 and 7-10 days respectively after kaolin injection using microarray technique. At the transcriptional level, the young rats showed overexpression of $>100$ genes involved in excitatory neurotransmission, protective mechanisms, and myelin production. Although adult rats showed elevated expression of inflammation mediating genes, they failed to show changes in genes involved in protective mechanism. These results indicate that there is an age dependant difference in gene expression profile of kaolin-induced hydrocephalus. (Funded by CIHR)

\section{Neuropathological study of a severe microcephaly associated with prenatal cocaine exposure}

\section{C.FALLET-BIANCO ${ }^{1}$, D. LUTON ${ }^{2}$, E. VUILLARD ${ }^{2}$, C. BOISSINOT ${ }^{2}$, A. KESROUANI ${ }^{2}$ E. JACQZ-AIGRAIN ${ }^{2}$, P. BLOT ${ }^{2}$, C. DAUMAS-DUPORT ${ }^{1}\left({ }^{1}\right.$ Department of Pathology, Hôpital Sainte-Anne, Paris, France, ${ }^{2}$ Hôpital Robert-Debre, Paris, France)}

The dramatic increase of cocaine abuse and its spread in Europe over the last decade is a serious social and medical problem. Recent estimates of the prevalence of cocaine use by pregnant women in different regions of North America range between $5,6 \%$ to $20 \%$. Recent imaging studies have disclosed an increased incidence of central nervous system lesions suggesting a disturbing effect of cocaine on brain development. Still human studies lack neuropathological data. Experimental studies have confirmed the involvement of cocaine in severe disturbances of neuronal migration and cortical development. We report a case of severe microcephaly in a cocaine-exposed fetus. The 34-year-old mother was a recreational drug-user including heroine and cocaine in the last eight years. Although she was undergoing replacement therapy, during her pregnancy, urine analysis 
revealed opiates, cocaine and its metabolites, demonstrating recent drug abuse. In relation with the poor predicted prognosis for this fetus, a termination of pregnancy was performed at 25 weeks. Neuropathological study confirmed the microcephaly and showed severe alterations of neurogenesis and gliogenesis associated with neuronal migration abnormalities strikingly similar to those described in animal models of cocaine gestational exposure. To the best of our knowledge, this case is the second reported neuropathological study of developmental brain lesions associated with fetal cocaine exposure.

\section{Untreated chronic hydrocephalus in the rat brain affects behavior and brain composition}

M.J. WILSON, T. ENNO and M.R. DEL BIGIO (Departments of Human Anatomy and Cell Science, and Pathology, University of Manitoba, Winnipeg, Canada)

Hydrocephalus is a common neurological condition in children wherein the ventricles of the brain enlarge due to impaired flow of the cerebrospinal fluid. This study was designed to understand the effects of chronic untreated hydrocephalus in rats. Three-week-old rats were injected with kaolin into the cisterna magna to induce hydrocephalus. Weight gain in hydrocephalic rats $(n=20)$ was significantly less than in controls $(n=10)$. Magnetic resonance imaging was performed on the rats at four and nine months postinjection. Ventricles enlarged gradually from four to nine months and were significantly enlarged in hydrocephalic rats $(41 \%$ of intracranial volume) compared to controls $(0.2 \%)$. Hydrocephalic rats exhibited significant memory impairment in the water maze, compromised agility on the rotarod, and impaired gait on open field observation and treadmill. Western blot analyses showed increased glial fibrillary acidic protein, decreased myelin basic protein, and decreased synaptophysin in the anterior cerebrum of hydrocephalic rats. Myelin-related enzyme activity in the fimbria increased as the ventricles enlarged, suggesting an increase in myelin turnover. These results show that changes in behavior and brain composition correlate with changes in ventricle size. [Funded by CIHR]

\section{Transneuronal atrophy in the lateral geniculate nucleus (LGN) neurons increases with optic nerve fiber loss}

Y.H. YUCEL ${ }^{1}$, Q. ZHANG ${ }^{1}$, P.L. KAUFMAN ${ }^{2}$, R.N.WEINREB ${ }^{3}$ and N. GUPTA (Departments of Ophthalmology and Laboratory Medicine and Pathobiology, St. Michael's Hospital, University of Toronto ${ }^{l}$; Department of Ophthalmology \& Visual Sciences, University of Wisconsin, Madison ${ }^{2}$; Department of Ophthalmology, University of California, San Diego ${ }^{3}$ )

Objective: To determine whether there is atrophy of target neurons in the LGN layers after varying degrees of chronic partial deafferentation of the optic nerve fibers by chronic intraocular pressure elevation, and its relationship to the degree of optic nerve damage. Methods: The left LGN of seven cynomolgus monkeys with chronic elevated eye pressure in the right eye and of five control monkeys were studied. In both groups, the same conditions of fixation, tissue processing, staining, and measurement were used. In each monkey, the left LGN target neurons in magnocellular layer 1 and parvocellular layers 4 and 6 , connected to the right experimental eye, were studied. Immunocytochemistry with antibody to parvalbumin was used to specifically label relay neurons connecting to the visual cortex. Cell body cross-sectional area of neurons was estimated using an unbiased point counting method. Results: The mean neuronal mean cross-sectional area in the layers connected to the experimental eye was significantly decreased in the experimental group compared to the control group. Mean neuronal area decreased as optic nerve fiber loss increased and this linear relationship was significant. Conclusion: Significant atrophic changes occur in surviving relay LGN neurons following partial deafferentation at the level of the optic nerve fibers, and the degree of atrophy appears to be dependent on the degree of optic nerve damage. This experimental model of chronic partial deafferentation may be relevant to understanding mechanisms underlying progressive transneuronal damage in neurodegenerative disease.

Supported in part by E.A. Baker Foundation of CNIB, Glaucoma Research Society of Canada, The Glaucoma Foundation, NY, NEI (EY02698), Joseph Drown Foundation.

\section{Neuropathological findings in a patient with a mutation in exon 10 of the pyruvate dehydrogenase E-1 alpha gene}

\section{J. MICHAUD, A. BLUMENTHAL, P. HUMPHREYS, P. JACOB, B.H. ROBINSON (Children's Hospital of Eastern Ontario, University of Ottawa)}

Human pyruvate dehydrogenase (PDH) complex deficiency is an inborn error of metabolism with a very heterogeneous clinical phenotype dominated by severe encephalopathy and lactic acidosis. We report the neuropathological findings associated with a 947C-T mutation in exon 10 of the PDH E-1 alpha gene providing a proline to leucine change. This eightmonth-old girl was born at term with severe lethargia, hypotonia and had seizure on her first day. Diagnostic imaging showed cerebral atrophy with enlargement of the ventricular system. Sequential clinical evaluations showed developmental delay, deafness and limited vision. The brain weighed $278 \mathrm{~g}$. There was severe atrophy of gyri, optic nerves, mammillary bodies and brain stem. The cerebellum was asymmetric with a punctiform depression in the left hemisphere. Histologically, multiple foci of leptomeningeal neuroglial heterotopias and dysplasia of the hippocampi and the inferior olivary and dentate nuclei were found in association with remote vascular-like lesions and a widespread neuronal loss. These findings, both degenerative and developmental in nature, point towards an early developmental occurrence and are similar to those reported in other cases of PDH E-1 alpha gene deficiency. The fetal occurrence illustrates the increased energy requirements of the developing brain and will complicate the implementation of potential therapy. 


\section{Immunohistochemical investigation of beta amyloid in the human brain associated with aging and non- dementia diseases}

S. HASHIMOTO, R. ITO, Y. HAYASHI, T. ITOH and T. SATOU (Second Department of Pathology, Kinki University School of Medicine, Osaka, Japan)

Although deposits of beta amyloid (Abeta) are considered to be profoundly related to aging, we examined their appearance in organic diseased brain (DB) samples taken from autopsy cases. Twenty-seven cases of various kinds of diseases which were nondementia and non-Alzheimer disease were represented. The immunohistochemical reactivity of A beta in these cases was evaluated in relation to age as well as age-matched nondiseased brain specimens (non-DB, 19 cases).

After fixation in $10 \%$ buffered formalin, $100 \mu \mathrm{m}$ thick sections from the frontal lobe and hippocampus were prepared with a microslicer, and then reacted with polyclonal antibodies for A beta. Positivity was quantitatively evaluated under a light microscope and statistically analyzed. In the DB cases, A beta was found to be increased in statistical correlation to age, but was not correlated to age in cases of non-DB. Our results suggest that A beta deposits are due to a multiplex of factors that arise from various disease processes as well as aging.

\section{The expression of cell cycle regulator proteins CDK4 and p16 in Alzheimer and non-Alzheimer dementias}

S.C. SUE, A.R. TAYLOR, L.C. ANG (Department of Pathology, London Health Sciences Centre and University of Western Ontario, London, Ontario)

Previous studies (Nagy et al, 1997, McShea et al, 1997) on the expression of numerous cell cycle control elements such as CDK4, p16, and various cyclins indicate an abortive attempt of terminally differentiated neurons to re-enter the cell cycle may be a critical event in Alzheimer-type changes. We examined the expression of CDK4 and p16 in the hippocampi from eight cases of Alzheimer's disease (AD), ten cases of Lewy body dementia (LBD), three cases of Pick's disease (PD), two cases of frontotemporal degeneration (FTD) and five cases of normal age-matched controls. The ages of cases ranged from 61 years to 93 years with a mean age of 78.2 years. Immunohistochemistry using the $\mathrm{ABC}$ method with antigen retrieval pretreatment was performed on paraffin blocks of the hippocampi. A subset of pyramidal neurons in the hippocampi was immunoreactive to CDK4 (Santa Cruz Biotech., CA, 1:100) and p16 (Santa Cruz Biotech., CA, 1:200) in all cases, including controls. As previously reported, the CDK4 immunolabels the neurofibrillary tangles and senile plaques associated with ADtype pathology. In the other dementias, the CDK4 highlights the Lewy bodies and the Pick bodies. The presence of CDK4, a protein that signals re-entry into the cell cycle, in non-AD dementias suggests the perturbations in cell cycle regulation is not unique to AD-type pathology.

\section{Pilomyxoid astrocytoma of the spinal cord}

C. RAO ${ }^{1}$, T. TIHAN ${ }^{2}$, P. BURGER ${ }^{2}$, R. FLAMHOLZ $Z^{3}, R$. KOTZEN ${ }^{3}$, R. TODOR ${ }^{1}$. ( ${ }^{1}$ State University of New York, Health Science Center, Brooklyn, ${ }^{2}$ The John Hopkins University School of Medicine, Baltimore, ${ }^{3}$ Long Island College Hospital, Brooklyn)

Pediatric astrocytomas with distinctive monomorphous pilomyxoid pattern in addition to some histological features of pilocytic astrocytoma (PA) were identified recently (Tihan et al. J Neuropathol Exp Neurol 1999;58(10):1061-1068). The reported tumors occurred at hypothalamus, optic chiasm, suprasellar region, temporal lobe and cerebellum and were associated with more aggressive outcome than most classical PAs. We report here, yet another location of this neoplasm in an eight-year-old boy who presented with lower back pain. MRI showed intramedullary spinal cord tumor from T9 to T12 with syringomyelia above and below the tumor. Total resection of the tumor was performed and histology revealed monomorphous, piloid tumor with abundance of myxoid areas. Focal perivascular arrangement of pilocytic cells was seen. Neither Rosenthal fibers nor eosinophilic granular bodies were identified.

Asa nosological niche for this tumor has yet to be carved, recognition and documentation of these neoplasms at various sites and close follow-up of the patients is important.

\section{The development of gliomas in a transgenic mice expressing a constitutively activated Ras}

P. SHANNON ${ }^{1}$, H. DING ${ }^{2}$, L. RONCARI ${ }^{2}, A . N A G Y^{3}$, and A. GUHA ${ }^{2}$ (The Toronto Western Hospital, Divisions of Neuropathology ${ }^{1}$ and Neurosurgery ${ }^{2}$ Surgery, and the Samuel Lunenfeld Research Institute ${ }^{3}$, Mount Sinai Hospital, Toronto, Ontario, Canada)

In a previous report we described the molecular and histological characteristics of astrocytic tumors arising in transgenic mice expressing oncogenic V(12)Ha-ras under the control of the GFAPpromoter (Ding et al, Cancer Res 2001 May 1;61(9):3826-36). In this report, we describe in more detail the patterns of pathology associated with these tumors, and in addition report the presence of an unusual type of cerebellar dysplasia characterised by focal hypoplasia of the cortical molecular layer. Transgenic mice unaffected by tumors display, in addition, a widespread astroglial hyperplasia that is morphologically distinct from reactive gliosis on the grounds of nuclear morphology. These findings are relevant to the identification of precursor lesions for human gliomas. 
14. Somatostatin receptors in medulloblastoma and ependymoma: Implications for diagnosis and therapy

J. GUYOTAT ${ }^{1}$, M. FEVRE-MONTANGE ${ }^{2}$, J. CHAMPIER ${ }^{2}$, I. CAMPOS ${ }^{3}$, Ph. BRET ${ }^{l}$, G. SAINT PIERRE ${ }^{l}$ and A. JOUVET ${ }^{l}$. $\left({ }^{1}\right.$ Hôpital Neurologique BP Lyon Montchat,Lyon. ${ }^{2}$ INSERM U.433, Faculté de Médecine Laennec, Lyon Cedex 08. ${ }^{3}$ Equipe d'accueil 3063, Faculté de Médecine, Saint-Etienne Cedex)

Expression of somatostatin receptors subtypes (sst1-sst5) was analyzed in 15 medulloblastomas (MB) and 28 ependymomas (EP) using semi-quantitative RT-PCR and immunohistochemistry. The five subtypes mRNA were variably expressed in each tumor.

The mRNAencoding sst2 was highly expressed in all tumors. This expression was higher in MB than in EP. The mRNA encoding sst1 was highly expressed only in EP. Immunohistochemistry revealed a predominant membrane localization of sst2A and a cytoplasmic labelling for sst 1 in MB. A low cytoplasmic labelling for stt2A and sst 1 was seen in EP. No correlation was observed between the expression of sst and age, location, histological grading, recurrence or survival.

An antiproliferative effect of somatostatin was shown in MB cell line and also in tumoral cells maintained in cultures. Furthermore, somatostatin scintigraphy showed clear uptake that agrees with MRI in 17 out of 23 performed investigations. These data open interesting prospects for the follow-up of these tumors.

\section{A rare complication of radiotherapy for craniopharyngioma - post radiation arteriopathy}

C. RAO ${ }^{1}$, J. RUIZ ${ }^{1}, J . L I^{2}$, R. ALEXIS ${ }^{2}\left({ }^{1}\right.$ State University of New York Health Science Center, Brooklyn, ${ }^{2}$ Long Island College Hospital , Brooklyn)

Craniopharyngioma is a benign but indolent tumor which because of its sheer location and anatomical relationship with hypothalamus and pituitary gland is associated with high morbidity due to endocrinopathy. Endocrinopathy thus, is a common complication, either preoperatively or postoperatively, whereas postradiation glioma, postradiation arteriopathy, malignant transformation of squamous epithelium and central pontine myelinolysis are rare complications. Five cases of occlusive arteriopathy following 2 to 6 years after last radiation therapy for craniopharyngioma are documented (Mori 1978, Nishizawa 1991, Sanford 1994, Bitzer 1995, Ishikawa 1997). We report here another case of radiation induced arteriopathy of right middle cerebral artery resulting in left hemiplegia. This 17year-old male had multiple surgeries and radiation treatment for craniopharyngioma at age four, seven and nine. He developed seizure disorder, panhypopituitarism, legal blindness, moderate mental retardation and was a resident of a chronic care facility where he developed left hemiparesis eight years after the last radiation.

At autopsy, the vessels of the Circle of Willis, especially right middle cerebral artery, revealed severe (80\%) occlusive arteriopathy, with a large infarct in its distribution territory.

\section{Tubular aggregates associated with ragged red fibers in a patient with chronic progressive external ophthalmoplegia plus}

E.S. JOHNSON, A. CHAN, I.M. MACDONALD and B. MARRIAGE (Departments of Laboratory Medicine and Pathology, Medical Genetics, and Ophthalmology, University of Alberta)

Recorded in this case report is the previously undocumented, unique concurrence of tubular aggregates with ragged red fibers in the setting of chronic progressive external ophthalmoplegia plus (CPEO). In addition to exercise induced myalgia, a 42year-old man presented with a clinical picture in keeping with a mitochondrial myopathy: ophthalmoplegia with left ptosis, progressive loss of hearing, mild generalized weakness, fatigue, and a severe hypertensive crisis. Confirming the diagnosis, a quadriceps muscle biopsy contained $2.5 \%$ ragged red fibers, in which there were ultrastructurally abnormal mitochondria with paracrystalline inclusions, and on Southern blot analysis demonstrated a 5 kilobase mitochondrial DNAdeletion spanning the ATPase 6 gene to the ND5 gene. Tubular aggregates, present in similar proportion, were found on electron microscopy to be accompanied by cylindrical spirals and abnormal tubulomembranous spirals. Other biopsy findings noted were marked type II predominance and nonspecific changes, including $12 \%$ of fibers with internal nuclei. The association of tubular aggregates and the accompanying tubulomembranous abnormalities in this patient's biopsy with a common mitochondrial deletion found in Kearns Sayre syndrome and CPEO is speculative, but does correspond with recent experimental observations of the presence of tubular aggregates in mice with mutant mitochondrial dysfunction (Cell, 1997, 89:93-103; Mech Ageing Dev, 2000, 114:89-99).

\section{Duchenne muscular dystrophy: A historical perspective}

VENITA JAY, MD, FRCPC (Division of Pathology, The

Hospital for Sick Children-University of Toronto)

While the eponym, Duchenne muscular dystrophy (DMD), is applied to the most common muscular dystrophy of childhood, Guillaume-Benjamin-Amand Duchenne (1806-1875) was not the first to describe the condition. The London physician, Edward Meryon, had described this entity in a family with four affected boys, ten years before Duchenne. But Duchenne provided a comprehensive account of the clinical and pathological features of the disease. Duchenne was a pioneer in applying electricity to the study of disease and introducing "muscle biopsy" into clinical practice. He taught himself microscopy and photography. In 1861, Duchenne described his first case of DMD. The association of two contradictory observations, paralysis and muscle hypertrophy, greatly perplexed him. In 1865, he developed his own "tissue punch" to clarify the muscle pathology. Because of the extreme muscle enlargement and fibrosis, he proposed the term, "Paralysie musculaire pseudohypertrophique". By 1870, Duchenne described three stages - feeble movements, apparent hypertrophy, and finally, paralysis.

He noted that it was "more prevalent in boys". His muscle biopsies revealed gradual accumulation of fibrous, and in later 
stages, fatty tissue. In 1871 , he was able to confirm that there was no neurogenic lesion in the cord. Duchenne's career was quite unorthodox. He was the physician at large in Paris, not affiliated with any one institution in particular, but welcome in all. When he died in 1875, Duchenne was recognized as an original medical scientist.

\section{Oxidative neuroglial toxicity in sepsis and HIV-1 encephalitis}

R.R. HAMMOND, J.X. WILSON, J. KORCOK, S. ISKANDER, J. CRUKLEY, J. NASSIF and A.W. GELB (London Health Sciences Centre, University of Western Ontario)

Mechanisms of neuroglial toxicity associated with HIV-1 encephalitis and sepsis remain unresolved but oxidative injury and resistance to it are felt to be important factors. Primary dissociated rat and human CNS tissue cultures are sensitive and reliable systems in which to study the effects of a variety of neurotoxins including HIV-1 gp120 and lipopolysaccharide/ interferon-gamma (LPS/IFN). Both culture formats are stable for many weeks, supporting mature neuronal and glial subpopulations. In vitro exposure to low level $(0.1-1 \mathrm{nM})$ recombinant gp-120 leads to astrocytosis and a simplification of neuronal dendrites mimicking some of the neuropathological consequences of HIV-1 infection in the human brain. Cultures can be pretreated with zinc or low dose ionizing radiation to augment antioxidant metallothionein expression and provide protection from oxidative stresses. Calcium channel blockers (nifedipine) are effective in protecting against gp120 associated neuronal damage. Similarly, LPS/IFN exposure leads to increased astroglial iNOS expression and impaired glutamate uptake, both of which can be mitigated by ascorbate supplementation. The cultures are flexible and reliable systems for studying neurotoxic sequelae of infectious and inflammatory states and the potential neuroprotective role of antioxidants therein. (Supported by grants from the Ontario HIV Treatment Foundation (RH) and the Natural Sciences \& Engineering Research Council of Canada (JXW))

\section{Positron emission tomography (PET) imaging of macrophage activation in SIV encephalitis}

\author{
C.A. WILEY, S. BISSEL, S. VENNETI, G. WANG, S. \\ CAPUANO, M. MURPHEY-CORB, M. PIATEK ${ }^{+}$, J.D. \\ LIFSON $^{+}$, B. LOPRESTI, C.A. MATHIS (University of \\ Pittsburgh, Pittsburgh, PA. ${ }^{+}$National Cancer Institute, \\ Fredrick, MD)
}

The radiolabelled ligan ([11C]-PK11195) binds to the peripheral benzodiazepine receptor (PBDR). Binding of [11C]PK11195 to primate macrophages and astrocytes was assessed in vitro. Because macrophages express high levels of this receptor, we examined the feasibility of using this ligand and 3D PET to follow macrophage infiltration in vivo during lentiviral encephalitis. Macaques were infected with the B670 strain of SIV and sacrificed from three months to two years postinoculation. Six SIV-infected macaques were imaged using [11C]-PK11195 followed by complete neuropathological examination. Two of the macaques had SIV encephalitis (with greater than $10^{7}$ copies of SIV RNAper mg brain tissue), one had acute and chronic infarcts and the others were without significant neuropathology. Chronic infarcts containing dense macrophage infiltration appeared as localized regions of high radioactivity on 3D PET. Macaques with SIV encephalitis showed a greater than two-fold increase in radioactivity throughout the CNS compared to macaques without encephalitis. Quantitative PET imaging may be useful in monitoring the progression of lentiviral mediated neurodegeneration and therapeutic interventions.

\section{Investigating the function of the prion protein homologue Doppel}

C. HAWKINS, I. RADOVANOVIC, L. MUYS and A. AGUZZI (Institute of Neuropathology, University Hospital Zurich and Department of Laboratory Medicine and Pathobiology, University of Toronto)

Doppel ( $\mathrm{Dpl})$ is a recently discovered protein encoded by the Prnd locus located $16 \mathrm{~kb}$ downstream of Prnp. It has $23 \%$ identity to the cellular prion protein $\left(\mathrm{PrP}^{\mathrm{C}}\right)$ and is involved in neuronal toxicity, especially in Purkinje cell degeneration in several lines of Prnp $^{0 / 0}$ mice.

This phenomenon is rescued by reintroduction of a Prnp allele, suggesting that $\mathrm{PrP}^{\mathrm{C}}$ prevents Dpl toxicity. But little is known about the mechanism by which Dpl mediates neuronal cell death or what its normal function may be. To investigate this, we have expressed Dpl, using an adenoviral-based vector, in organotypic brain slices from P2 and P5 Prnp ${ }^{0 / 0}$ mice. We found that mature neurons and glia undergo cell death in the Dplinfected slices but that the nestin-positive, periventricular cells seem to be immune to this effect. Further, neurite outgrowth in the remaining cells is disrupted. Interestingly, glial tumour cell lines as well as cerebellar granular cells appear to be susceptible to the cell death effects of Dpl while cell lines derived from mesenchymal and epithelial tissues are resistant. Ongoing studies are aimed at: 1) examining the downstream pathways mediating the cell death effect of $\mathrm{Dpl}$ and, 2) using an in vitro cerebellar precursor differentiation model, investigating the potential of $\mathrm{Dpl}$ to inhibit differentiation of neuroepithelial precursor cells and its effect on neurite outgrowth. 


\section{Titles of Diagnostic Case Presentations}

1. Superficial siderosis; presumably "idiopathic"

S. ALBRECHT (Department of Pathology, Sir Mortimor B. Davis Jewish General Hospital and McGill University, Montreal)

2. Thrombophilia and dementia in a young man heterozygous for both, Factor $V$ Leiden and methylenetetrahydrofolate reductase variant

M. URSELL ${ }^{1}$, J.M. BILBAO ${ }^{1}$, B. YOUNG ${ }^{1}$, S.E. BLACK ${ }^{2}$ $\left({ }^{1}\right.$ Department of Anatomic Pathology and ${ }^{2}$ Neurology, Sunnybrook \& Women's College Health Sciences Centre, University of Toronto)

3. Extra-osseous myxoid chondrosarcoma (embryonal type) from the dura

K. MEAGHER-VILLEMURE ${ }^{1}$, S. GEBHARD ${ }^{1}$, D. CHAREST ${ }^{2}$, J-G. VILLEMURE ${ }^{2}$ (Institut Universitaire de Pathologie ${ }^{1}$, Service de Neurochirurgie ${ }^{2}$, Centre Hospitalier Universitaire Vaudois, Lausanne, Switzerland)

\section{Cerebellar liponeurocytoma with atypical features}

J.P. ROSSITER and D. LEE (Department of Pathology and Division of Hematology/Oncology ${ }^{1}$, Queen's University and Kingston General Hospital, Kingston, Ontario)

5. Left parasagittal inflammatory pseudotumor, consistent with Rosai Dorfman disease

C. MASCOTT ${ }^{1}$, M.B. BHATTACHARJEE (Departments of Neurosurgery ${ }^{1}$ and Pathology \& Laboratory Medicine, Tulane University Health Science Center, New Orleans, LA)
6. Acute necrotizing encephalopathy of childhood W.L. STEFANEK ${ }^{1}$, D. GEORGE ${ }^{1}$ and C. TREVENEN ${ }^{2}$ (Department of Pathology, Foothills Medical Centre ${ }^{1}$ and Alberta Children's Hospital', Calgary, Alberta)

7. Meningioma with focal granulomatous inflammation (PCR positive for Mycobacterium tuberculosis)

G. MEDVEDEV and I.R.A. MACKENZIE (Department of Pathology \& Laboratory Medicine, Division of Neuropathology, Vancouver Hospital \& Health Sciences Centre and University of British Columbia)

8. Rabies encephalomyelitis, paralytic type

Y. ROBITAILLE (Dept. of Pathology \& Cell Biology, University of Montreal, Ste-Justine Hospital, Montreal, Quebec)

9. Lymphocytic vasculitis consistent with primary angiitis of the CNS

J. FERREIRA (Department of Pathology, MaisonneuveRosemont Hospital, University of Montreal)

10. Jakob-Creutzfeldt disease

A.H. KOEPPEN, A.C. DICKSON (Neurology and Research Services, VA Medical Center, Albany, New York 12208 USA, and Albany Medical College) 